in developing countries such as Papua New Guinea, Fiji, the Solomon Islands, Vanuatu, and Indonesia where nurses receive little post basic education, have limited opportunities to review the evidence for practice in disaster and emergency nursing, yet are regularly required to support the multidisciplinary disaster health team without essential support from the nursing research community. The network is essentially web-based and consists of a translational research approach via a network grid of researchers in response to a disaster event. Researchers from neighboring countries not clinically involved in the event respond by discovering and accessing data, analysing and reporting through a portal that enables timely reporting for discussion, publication, e-learning and dissemination of contemporary disaster nursing practices. This paper will report on the development of the network and its nexus with the WADEM Nursing Research Committee.

Prehosp Disaster Med 2011;26(Suppl. 1):s15-s16

doi:10.1017/S1049023X1100063X

(A58) Guidelines for Reports on Health Crises and Critical Health Events - A Tool for the Development of Disaster Medicine Research

P. Kulling, ${ }^{1}$ M. Birnbaum, ${ }^{2}$ V. Murray, ${ }^{3}$

G. Rockenschaub ${ }^{4}$

1. Emerit Directorate General for Health and Consumers, Stockholm, Sweden

2. Madison, United States of America

3. London, United Kingdom

4. Copenhagen, Denmark

To develop a common structure for reports on health crises and critical health events guidelines have recently been published (Kulling et al 2010). They try to capture the experiences gained and for promoting a standardized methodology for sharing results and lessons. If future reporting follows common standards, then the documented findings would be comparable and could be used to learn and apply lessons within an individual field of activity and to apply those lessons learned also to other related preparedness activities. It could also facilitate the implementation of joint activities and joint reports involving different sectors. The development of this proposed method for common reporting on health crises and critical health events has been derived mainly from the following processes: (i) Health Disaster Management: Guidelines for Evaluation and Research in the Utstein Style; (ii) the Swedish Disaster Medicine Study Organization (KAMEDO); (iii) the Swedish Emergency Management Agency network of observers; (iv) the WHO Regional Office for Europe project (supported by the EU Health Programme) 'Support Health Security, Preparedness Planning and Crisis Management in the EU, EU accession and neighboring (ENP) countries' including expert consultations. The guidelines include the following headlines: Title, Preface, Authors, Executive Summary, Introduction/Material/Methodology, PreEvent Status (Background, Preparedness, Hazard(s)involved, Risks, Vulnerability, Resilience), Health Crises and Critical Health Events (Damage, Consequences of Damage (Changes in Functions/Disturbances), Responses(ReliefResponses, Recovery Responses), Development, Discussion, Lessons Identified and Actions Recommended, Conclusions, References, Appendices,
Keywords, Index, Abbreviations. Pilot testing is suggested followed by an extensive review process. The guidelines should be supplemented further with determinants and indicators when the guidelines are used for in depth reporting to evaluate crisis response operations. Reference Kulling P, Birnbaum M, Murray V, Rockenschaub G. Guidelines for Reports on Health Crises and Critical Health Events.

Prehosp Disaster Med 2011;26(Suppl. 1):s16 doi:10.1017/S1049023X11000653

\section{(A59) Advent of the Confide Guidelines for Disaster Medicine Reporting D. A. Bradt, ${ }^{1}$ P. Aitken ${ }^{2}$}

1. Center for Refugee and Disaster Response, Baltimore, United States of America

2. Anton Breinl Centre for Public Health and Tropical Medicine, Townsville, Australia

Disaster reports are common in the literature. Accurate and complete reporting assists readers and researchers in developing best practices. There have been notable efforts, such as the Utstein Template, to standardize the language of disasters and promote consistent use of definitions. However, case reports are complicated by the presence of four dominant types recognized in the literature. Moreover, the disciplines of medicine, public health, and disaster management differ in origins, definitions, research paradigms, and tools of evidence-based decision-making. Finally, biomedical journal guidelines for authors writing disaster case reports have lacked the rigor of standards associated with observational studies (2007 STROBE statement, 2010 MOOSE statement), or with randomized controlled trials (1996 CONSORT statement, 1999 QUORUM statement). This paper examines current efforts to intensify the rigor of future disaster case reports through uniform reporting requirements for authors. The initial workgroup comprised editorial board members of two biomedical journals indexed in the US National Library of Medicine-Prehospital and Disaster Medicine, and Emergency Medicine Australasia. The workgroup members self-selected based on extensive disaster field experience as technical advisor with governmental, non-governmental, Red Cross and UN agencies. The workgroup identified key information needed to understand the context, structure, process, and outcome/impact of disaster field interventions. Then, the workgroup organized this information in thematic domains. Consensus guidelines emerged for Reports of Field Interventions in Disasters and Emergencies (CONFIDE). The CONFIDE Statement addresses 16 keys areas within seven domains, including: field authorization for access, field logistics, initial assessment, clinical epidemiology, and funding. The Statement was first published in December 2010, at which time, the authors began integrating the guidelines with biomedical journal instructions for authors. The paper details current efforts to broaden editorial acceptance of the guidelines, implications for future authors, and potential benefits to the disaster medicine community.

Prehosp Disaster Med 2011;26(Suppl. 1):s16

doi:10.1017/S1049023X11000665 